\title{
Enhanced NLO response in BODIPY-coumarin hybrids: density functional theory approach
}

\author{
YOGESH ERANDE and NAGAIYAN SEKAR* \\ Department of Dyestuff Technology, Institute of Chemical Technology, N. P. Marg, Matunga, Mumbai, \\ Maharashtra 400 019, India \\ E-mail: n.sekar@ictmumbai.edu.in; nethi.sekar@gmail.com
}

MS received 24 April 2017; revised 9 June 2017; accepted 20 June 2017; published online 27 July 2017

\begin{abstract}
We have thoroughly investigated the first, second and third polarizability characteristics of four hybrid chromophores by spectroscopic and computational methods. B3LYP, CAMB3LYP and BHandHLYP functionals in combination with $6-311+\mathrm{G}(\mathrm{d}, \mathrm{p})$ basis set were used to evaluate the polarizability and hyperpolarizability characteristics of these chromophores. Generalized Mulliken Hush analysis and frontier molecular orbital electronic distribution images of chromophores obtained from Density functional theory computation has established the charge transfer characteristics of these hybrid chromophores. On the basis of charge transfer characteristic, these red absorbing and NIR emissive chromophores possess high nonlinear optical response. Comparison of isolated units with their analogous hybrid chromophores shows that fusion of coumarin with BODIPY enhances the nonlinear optical response.
\end{abstract}

Keywords. Coumarin fused BODIPY; CAM-B3LYP; hyperpolarizability; push-pull; TDDFT.

\section{Introduction}

Investigation of nonlinear optical (NLO) properties of BODIPY and related chromophores is an area of recent interest and very little work is done in this area. This inspired us to study the linear and nonlinear property of coumarin fused BODIPY hybrid chromophores. Convincing photophysical properties and their tunability have made BODIPY dyes, the molecules of choice in functional applications. ${ }^{1-3}$ The BODIPY dyes have been investigated in optoelectronic gates ${ }^{4}$ and they have been shown to have two photon and multiphoton absorption properties. ${ }^{5}$ Jacquemin and co-workers have done the theoretical investigation of optical signatures of BODIPY dyes. ${ }^{6-9}$ Studies in NLO properties of the BODIPY dyes have recently attracted attention. ${ }^{10-13}$ The property of two photon excitation of BODIPY dyes has been used in imaging and telecommunication applications. ${ }^{2,14-16}$ Optoelectronic properties of polyacetylenes containing BODIPY pendants with different connectivity have been examined. ${ }^{17}$

\footnotetext{
*For correspondence
}

Quantum mechanical studies have given general guidelines in the design of NLOphoric materials, and it has been known that an asymmetric distribution of the $\pi$ electrons within a conjugated push-pull molecule will give rise to an NLO response. Such an understanding has been qualitatively and quantitatively evolved in Oudar's two-state model, which has been widely used as the design tool at the conceptual level in realizing organic NLO molecules, having a large hyperpolarizability $(\beta) .{ }^{18-20}$ This model propounds that $\beta$ is proportional to the charge transfer dipole moment $\Delta \mu_{\mathrm{eg}}$ as well as the square of the transition dipole moment, $\mu_{\mathrm{eg}}$. Also, there exists an inverse proportionality between $\beta$ and the energy gap between the ground and first excited states, $\mathrm{E}_{\mathrm{eg}} \cdot{ }^{21}$

$\beta \propto \frac{\mu_{g e} \Delta \mu_{g e}}{\left(\Delta E_{e g}\right)^{2}}$

The fact that $\beta$ has an intrinsic relation with the charge transfer excited states at the molecular level is established by this model ${ }^{22,23}$ and has been widely verified. It has also been established that, just an increase in the strength of donor and acceptor in pushpull chromophores alone is not the only necessary 


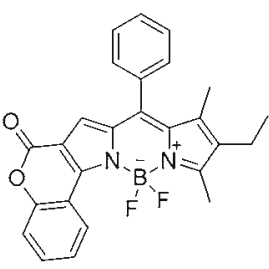

1

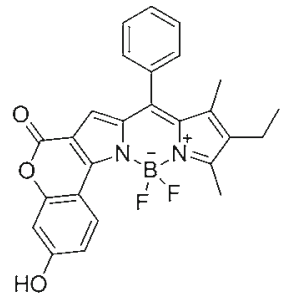

2

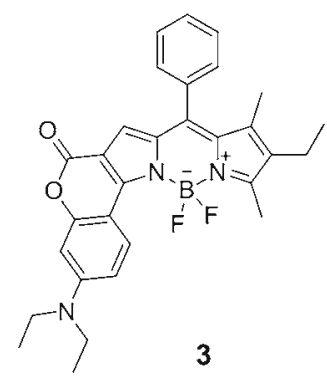

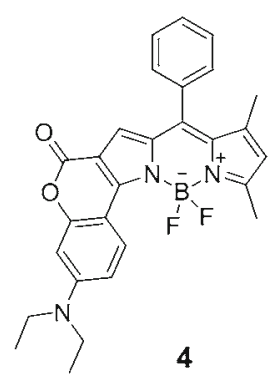

Figure 1. Structures of hybrid chromophores

aspect; a fully delocalized ground and excited states are also important. ${ }^{24,25}$ An optimum second order nonlinear optical response can be achieved, where there is an appreciable overlap of the wave functions that define the two states. ${ }^{26-28}$ Multipolar transitions ${ }^{29}$ and transitions involving the other excited states are also known to contribute to the first order hyperpolarizability response. ${ }^{30-33}$

Also, organic compounds having the nonlinearity caused by intramolecular charge transfer (ICT) in the nonresonant region are the candidates with large second order hyperpolarizability $(\gamma){ }^{34}$ The $\gamma$ values are only composed of contributions from the redistribution of the electron cloud which is a consequence of ICT phenomenon in the push-pull type organic $\pi$-conjugated molecular systems. Such materials are expected to play a key role in all optical switching devices, since their optical properties can be controlled by light. But due to the higher order of the nonlinearity and the requirement of investigation of femtosecond non-resonant dynamics, the third order optical nonlinearity of molecules possessing large ICT are lagging behind compared to second order nonlinearity as far as the studies related to efficiency is concerned. ${ }^{34,35}$ But here we have addressed both second and third order optical nonlinearity with the help of spectroscopic properties and on theoretical basis. The former is by using absorption spectra and latter by comprehensive computational optimization using three different hybrid functionals viz., B3LYP/6-311+G(d, p), CAM-B3LYP/6-311+G(d, p) and BHandHLYP/6$311+\mathrm{G}(\mathrm{d}, \mathrm{p})$.

Conceptually the molecules having red-shifted absorption and multipolar transitions should possess high nonlinear optical characteristics. In this paper, we have selected the coumarin-BODIPY hybrids (Figure 1) for nonlinear optical studies which are known to show large Stokes shift combining some exceptional properties of coumarin and BODIPY fluorophores. ${ }^{36}$ Two dyes (3 and 4) have NIR absorption while the other dyes (1 and 2) do not have red absorption.
In accordance with the designing guidelines from Oudar's two level model, the coumarin-fused BODIPY have $-\mathrm{OH}$ or $\mathrm{N}, \mathrm{N}$-diethyl as donors and quaternary $\mathbf{N}$ from quasi-aromatic ring as acceptor, linked by extensive $\pi$-conjugation over the annulated path of hybrid molecules. So, these are ICT-prone molecules and with respect to their polarized ground and charge transfer excited states, the maximization of $\beta$ can be realized by increasing the product of $\mu_{\mathrm{eg}} \Delta \mu_{\mathrm{eg}}$ or by decrease in ICT transition energy $\Delta \mathrm{E}$. Due to ICT, these molecules possess intense absorption bands in visible region and the electronic structure of these dyes can be an approximation of the combination between two limiting neutral and cyanine-type resonance forms. The relative contributions of these forms to NLO properties are dependent on the nature of the $\mathrm{D}$ and $\mathrm{A}$ groups and on the nature and length of the $\pi$-conjugation path. By virtue of the Oudar's two state approximation model, the maximum $\beta$ value can be achieved by appropriate combination of $\pi$-conjugation chain and D-A strength to have optimum bond length alternation (BLA) and bond order alternation (BOA), since even small changes in their values lead to dramatic changes in linear and NLO properties. ${ }^{37}$ BOA directly assesses the molecular electronic structure while BLA presents a measure of the molecular geometric structure, that is often but not always a good reflector of the electronic structure, ${ }^{37}$ and hence we studied both to examine their effect on NLO properties of coumarin-BODIPY hybrids.

Concerning the efficiency of charge transfer process, as the hybrids are not typical styryl-type conventional $D-\pi-A$ NLOphores, the Generalized Mulliken Hush $(\mathrm{GMH})$ analysis was done for the molecules. As there is a large $\pi$-conjugation over annulated path between $\mathrm{D}$ and $\mathrm{A}$, calculation of the effective D-A distance and strength of coupling are worth for understanding the nature of coupling, which is responsible for NLO performance of the molecules. ${ }^{38}$ On the basis of $\mathrm{GMH}$ scheme, the coupling strength $\mathrm{H}_{\mathrm{DA}}$ between ground state and charge transfer excited state is calculated. 
Table 1. Photophysical properties of dyes $\mathbf{1}$ to $\mathbf{4}$ in dichloromethane.

\begin{tabular}{|c|c|c|c|c|c|c|c|c|c|}
\hline Dye & $\begin{array}{l}\lambda_{\mathrm{abs}}^{[\mathrm{a}]} \\
(\mathrm{nm})\end{array}$ & $\varepsilon^{[\mathrm{b}]}$ & $\begin{array}{l}\mathrm{FWHM}^{[\mathrm{c}]} \\
\quad\left(\mathrm{cm}^{-1}\right)\end{array}$ & $f^{[\mathrm{d}]}$ & $\begin{array}{l}\lambda_{\mathrm{em}}^{[\mathrm{e}]} \\
(\mathrm{nm})\end{array}$ & $\begin{array}{l}\text { Stokes } \\
(\mathrm{nm})\end{array}$ & $\begin{array}{l}\text { shift } \\
\left(\mathrm{cm}^{-1}\right)\end{array}$ & $\Phi e m^{[\mathrm{f}]}$ & $\begin{array}{c}\mu_{\text {ge }}(\text { abs })^{[\mathrm{g}]} \\
\text { (Debye) }\end{array}$ \\
\hline 1 & 516 & 36000 & 2733 & 0.72 & 546 & 30 & 1065 & 0.66 & 7.03 \\
\hline 2 & 530 & 46200 & 1985 & 0.69 & 567 & 37 & 1231 & 0.64 & 6.88 \\
\hline 3 & 602 & 59400 & 2409 & 0.69 & 697 & 95 & 2264 & 0.41 & 9.16 \\
\hline 4 & 606 & 59100 & 2353 & 0.58 & 706 & 100 & 2337 & 0.31 & 9.06 \\
\hline
\end{tabular}

[a] Experimental absorption maximum; [b] Molar extinction coefficient at absorption maximum $\mathrm{M}^{-1} \mathrm{~cm}^{-1}$; [c] Full width at half absorption maximum; [d] Oscillator strength; [e] Experimental emission maximum; [f] Fluorescence quantum yield; [g] Transition dipole moment.

\section{Experimental}

\subsection{Materials and methods}

The ground state $\left(\mathrm{S}_{0}\right)$ geometries of the compounds $\mathbf{1 - 4}$ were optimized in the gas phase using Density Functional Theory (DFT). ${ }^{39}$ The popular hybrid functional B3LYP was used, which combines Becke's three parameter exchange functional (B3) ${ }^{40}$ with the nonlocal correlation functional by Lee, Yang, and Parr (LYP). ${ }^{41}$ All the atoms were treated with 6-311+ G (d,p) basis set, which seems to be sufficient for the type of molecules involved. ${ }^{42}$ The validity of the structures as local minima on potential energy surface was verified with vibrational analysis and confirmed that they are with no imaginary frequencies. The first and second order hyperpolarizabilities were calculated using the optimized geometry. At the DFT level, several exchange-correlation functionals were employed viz., B3LYP (generalized gradient approximationGGA), CAM-B3LYP (long range corrected hybrid functional with 19 to $65 \%$ HF contribution) and BHandHLYP (medium range electron correlations hybrid functional with a fixed $50 \%$ $\mathrm{HF})$ using 6-311+G(d,p) basis set. All the computations were carried out in solvent environment using the Polarizable Continuum Model (PCM). ${ }^{43}$ Gaussian 09 program $^{44}$ was used for all the DFT and TDDFT computations and the results were visualized with Gauss View 5.0. ${ }^{45}$

The NLO properties of the molecules are defined by parameters like $\alpha_{\mathrm{CT}}, \beta_{\mathrm{CT}}, \beta_{0}$, and $\gamma$. These parameters were obtained by computational method and from absorption spectroscopy. ICT characteristics of the dyes $\mathbf{1}$ to $\mathbf{4}$ were studied with the help of GMH analysis and bond BLA studies, which were evaluated from optimized geometries of the dyes with B3LYP functional and $6-311+\mathrm{G}(\mathrm{d}, \mathrm{p})$ basis set at ground state and excited state geometries. These geometries were obtained using TDDFT at the same level of theory.

\section{Result and Discussion}

\subsection{Photophysical properties}

The absorption and emission data of these coumarinfused BODIPY hybrid (CB) chromophores in dichloromethane are known in the literature ${ }^{36}$ which are summarized in Table 1. Dyes $\mathbf{1}$ and $\mathbf{2}$ have absorption maxima at $516 \mathrm{~nm}$ and $530 \mathrm{~nm}$, respectively while dyes 3 and $\mathbf{4}$ have absorption in the red region viz., $602 \mathrm{~nm}$ and $606 \mathrm{~nm}$, respectively, which is due to the presence of N,N-diethylamino group as strong donor on the coumarin part of chromophores. Emission maxima of dyes $\mathbf{3}$ and $\mathbf{4}$ have an appreciable red shift compared to dyes $\mathbf{1}$ and $\mathbf{2}$ with a Stokes shift ranging $100 \mathrm{~nm}$. The large Stokes shift for dyes $\mathbf{3}$ and $\mathbf{4}$ is attributed to their strong ICT character, because of N,N-diethylamino donor group at the place of hydroxyl moiety. From the absorption measurements, the transition dipole moments $\left(\mu_{\mathrm{ge}}\right)$ for all the chromophores were also determined; dyes 3 and 4 possess higher values than dyes $\mathbf{1}$ and $\mathbf{2}$ (Table 1). These values were used to determine the spectroscopic NLO properties and charge transfer characteristics of the chromophores. The oscillator strength (f) and transition dipole moment $\left(\mu_{\mathrm{ge}}\right)$ were calculated by using following equations: ${ }^{46,47}$

$$
\begin{aligned}
& f=\frac{4.32 \times 10^{-9}}{n} \int \varepsilon(v) d v \\
& \mu_{\mathrm{eg}}^{2}=\frac{f}{4.72 \times 10^{-7} X \bar{v}}
\end{aligned}
$$

where, $\varepsilon$ is the molar absorptivity $\left(\mathrm{L} \mathrm{mol}^{-1} \mathrm{~cm}^{-1}\right), v$ or $\bar{v}$ represents the frequency in wavenumber $\left(\mathrm{cm}^{-1}\right)$ of the corresponding absorption maximum. The photophysical properties of these chromophores are summarized in Table 1.

As mentioned earlier the charge transfer characteristics of these chromophores were established by GMH analysis, since these characteristics have great effect on NLO properties of push-pull type chromophores. The two state GMH analysis of intramolecular charge transfer (ICT) can be presented as follows: ${ }^{48}$

$\Delta \mu_{a b}^{2}=\Delta \mu_{g e}^{2}+4 \mu_{g e}^{2}$ 
Table 2. Charge transfer characteristics of dyes $\mathbf{1}$ to 4.

\begin{tabular}{cccccccccc}
\hline Dye & $\mathrm{IAC}^{[\mathrm{a}]} \mathrm{X} 10^{8}$ & $f^{[\mathrm{b}]}$ & $\begin{array}{c}\mu_{\mathrm{ge}}^{[\mathrm{g}]} \\
\text { Debye }\end{array}$ & $\begin{array}{c}\Delta \mu_{\mathrm{CT}}^{[\mathrm{d}]} \\
\text { Debye }\end{array}$ & $\begin{array}{c}\Delta \mu_{g e}^{D^{[e]}} \\
\text { Debye }\end{array}$ & $\begin{array}{c}\mathrm{C}_{\mathrm{b}}^{[\mathrm{f}]} \\
\mathrm{cm}^{-2}\end{array}$ & $\begin{array}{c}\mathrm{H}_{\mathrm{DA}}^{[\mathrm{g}]} \\
\mathrm{cm}^{-1}\end{array}$ & $\begin{array}{c}\mathrm{R}_{\mathrm{DA}}^{[\mathrm{h}]} \\
\AA\end{array}$ \\
\hline 1 & 1.04 & 0.72 & 7.03 & 0.70 & 14.09 & 0.48 & 9678 & 2.94 \\
2 & 0.97 & 0.69 & 6.88 & 0.70 & 13.78 & 0.47 & 9422 & 2.88 \\
3 & 1.52 & 0.69 & 9.16 & 0.04 & 18.33 & 0.50 & 8306 & 3.82 \\
4 & 1.47 & 0.58 & 9.06 & 0.30 & 18.13 & 0.49 & 8250 & 3.78 \\
\hline
\end{tabular}

[a] Integrated area of absorption coefficient; [b] Oscillator strength; [c] Transition dipole moment; [d] Difference between ground and excited state dipole moments; [e] Dipole moment change between the diabatic states; [f] Degree of delocalization; [g] Electronic coupling matrix/Strength of electronic coupling between the ground $\left(\mathrm{S}_{0}\right)$ and charge transfer excited states; [h] Donor-acceptor separation.

where, $\mu_{\mathrm{ge}}$ was obtained by eq. $3, \Delta \mu_{\mathrm{ge}}$ is the dipole moment difference between ground and excited states. The electronic coupling between D and A groups is an important factor in controlling the charge transfer for D-A push-pull systems. ${ }^{49}$ Therefore, it is necessary to determine the electronic coupling matrix elements $\left(\mathrm{H}_{\mathrm{ab}}\right)$ in order to understand the behavior of long distance charge transfer ( $a$ and $b$ refer to the initial and final diabetic states). In adiabatic state, the coupling matrix element of D- $\pi$-A systems can be denoted as $\mathrm{H}_{\mathrm{DA}}$. The diabatic states are considered as charge-localized valence bond structures, which characterize the ground and excited states in the charge transfer process. While the adiabatic states are assumed to be composed of these three diabatic states i.e., a donor ground state (GS), a donor locally excited state (LE), and a charge transfer state $(\mathrm{CT})$, which transfers the charge from donor to the acceptor. The electronic coupling matrix $\left(H_{D A}\right)$ and fractional degree of localization of the excess charge $\left(C_{b}^{2}\right)$ for the diabatic states can be described by following equations:

$$
\begin{aligned}
& H_{D A}=\frac{\Delta \mathrm{E}_{\mathrm{ge}} \cdot \mu_{\mathrm{ge}}}{\Delta \mu_{\mathrm{ab}}} \\
& C_{b}^{2}=\frac{1}{2}\left(1-\sqrt{\frac{\Delta \mu_{g e}^{2}}{\Delta \mu_{g e}^{2}+4 \mu_{g e}^{2}}}\right)
\end{aligned}
$$

Within the two level approximation, in eq. 5, the extent of donor-acceptor electronic coupling $\left(\mathrm{H}_{\mathrm{DA}}\right)$ between the ground and charge transfer excited states is correlated with the vertical excitation energy $\left(\Delta \mathrm{E}_{\mathrm{ge}}\right)$, the difference between the adiabatic dipole moments of the ground and excited states $\left(\Delta \mu_{\mathrm{ge}}\right)$, and the difference in diabatic state dipole moments and the transition dipole moments $\left(\Delta \mu_{\mathrm{ge}}^{\mathrm{D}}\right)$, by the GMH model, ${ }^{49-51}$ as in eq. 7 .

$$
H_{D A}=\frac{\Delta \mathrm{E}_{\mathrm{ge}} \mu_{\mathrm{ge}}}{\Delta \mu_{\mathrm{ge}}^{\mathrm{D}}}=\frac{\Delta \mathrm{E}_{\mathrm{ge}} \cdot \mu_{\mathrm{ge}}}{\sqrt{\Delta \mu_{g e}^{2}+4 \mu_{g e}^{2}}}
$$

The extent of charge separation in the charge transfer process from donor to acceptor through $\pi$-bridge can be determined by eq. $8 .^{50,52}$

$R_{D A}=2.06 \times 10^{-2} \frac{\sqrt{\Delta \mathrm{E}_{\mathrm{ge}} \varepsilon_{\max } \Delta \nu_{1 / 2}}}{\mathrm{H}_{\mathrm{DA}}}$

where, $R_{D A}$ is the separation distance between the centroids of the donor and acceptor orbitals $(\AA), \Delta E_{g e}$ is vertical excitation energy $\left(\mathrm{cm}^{-1}\right), \varepsilon_{\max }$ is molar absorptivity $\left(\mathrm{M}^{-1} \mathrm{~cm}^{-1}\right), \Delta v_{1 / 2}$ is bandwidth $\left(\mathrm{cm}^{-1}\right)$ and $H_{D A}$ is donor-acceptor electronic coupling matrix $\left(\mathrm{cm}^{-1}\right)$. The GMH characteristics are calculated using above equations and summarized in Table 2 . When $C_{b}^{2}$ is zero then it is total delocalization and when it is unity, then it is called total localization of the charge. The values of $C_{b}^{2}$ for all chromophores in all the solvents are $\approx 0.5$, indicating that there is high degree of delocalization which supports the ICT character of all the chromophores in DCM. The $\mathrm{R}_{\mathrm{DA}}$ values of dyes $\mathbf{3}$ and $\mathbf{4}$ are more than those for the dyes $\mathbf{1}$ and $\mathbf{2}$, and hence, the corresponding $\mathrm{H}_{\mathrm{DA}}$ values are less, since the separation distance increases as the donor-acceptor electronic coupling strength decreases.

\subsection{Nonlinear optical properties}

From GMH analysis it is confirmed that there is charge transfer in the excited state which makes the basis that these molecules can be studied as nonlinear optical chromophores. The linear and nonlinear optical properties of these chromophores were investigated by both spectroscopic and theoretical approaches by TDDFT calculations.

The BLA and BOA values are related to the first order hyperpolarizability values, since geometry and electronic structure of chromophores play important roles in determining its hyperpolarizability. The $\pi$-conjugated 


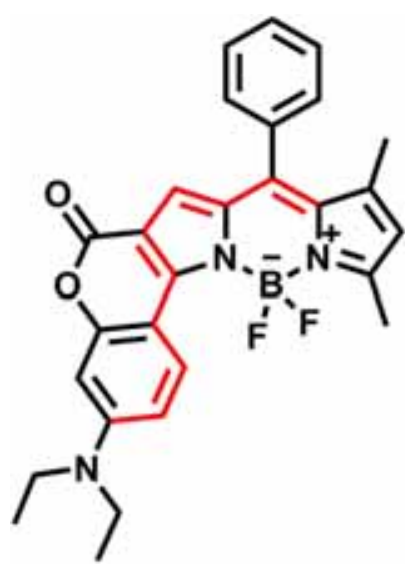

Figure 2. Structure showing red colored $\pi$-conjugated path used to calculate BLA/BOA.

Table 3. BLA and BOA values of dyes $\mathbf{1}$ to $\mathbf{4}$ at GS and ES; and CS for 3 .

\begin{tabular}{lcrllllll}
\hline Dye & \multicolumn{3}{c}{ BLA } & & & \multicolumn{3}{c}{ BOA } \\
\cline { 2 - 3 } \cline { 6 - 7 } & GS & ES & CS & & GS & ES & CS \\
\hline 1 & 0.015 & -0.011 & & & -0.017 & 0.121 & \\
2 & 0.01 & -0.007 & & & 0.014 & 0.121 & \\
3 & -0.003 & 0.006 & 0.009 & & 0.088 & 0.058 & 0.021 \\
4 & -0.006 & 0.007 & & & 0.097 & 0.052 & \\
\hline
\end{tabular}

(Crystal Structure, CS was obtained from the crystal structure which is available ${ }^{36}$ for only dye $\mathbf{3}$ )

path used to calculate BLA/BOA values is shown in Figure 2.

The excited state $\pi_{\mathrm{C}=\mathrm{C}}^{*}$ bond length and ground state $\pi_{\mathrm{C}=\mathrm{C}}$ bond length are obtained from the corresponding excited state and ground state optimized geometry of molecule at B3LYP/6-311+g(d, p) level in dichloromethane solvent.

Optimized NLO responses for the push-pull chromophores can be achieved by distorting the conjugation from polyene-like (unperturbed) structure towards the partially ionic cyanine-like (fully delocalized) structure. The distortion can be controlled by adjusting the strength of D and A groups. Hence, BLA and BOA of the D- $\pi$-A conjugated systems for the dyes 1-4 in $S_{0}$ (GS) and $S_{1}$ (ES) states were determined using their respective geometries obtained from TDDFT optimization and summarized in Table 3. More the BLA in the $\pi$-conjugated system higher is the charge perturbation, which leads to an effective charge transfer between D and A groups that influences the NLO behavior of the chromophores. The BLA values for dyes $\mathbf{1}$ to $\mathbf{4}$ are very close to zero indicating that the molecular structure is approaching to cyanine limit $(\mathrm{BLA}=0)$ from polyene limit (BLA $\approx \pm 0.1$ ). ${ }^{31}$ BOA values for all the dyes are close to zero, suggesting that the molecular structure is approaching to cyanine limit $(\mathrm{BOA}=0)$ from polyene limit (BOA $\approx \pm 0.6$ ). Further, for representative dye 3, the theoretical BLA/BOA values are supported with the same obtained from X-ray characterization results. ${ }^{36}$ From the crystal structure of dye $\mathbf{3}$, the bond lengths values the BLA (0.009) and BOA (0.021) values (Table 3 ) are calculated and it gives concrete evidence from the theoretical calculations that the corresponding molecular arrangement is approaching towards cyanine limit $(\mathrm{BLA} / \mathrm{BOA}=0)$. As the BOA originates from molecular electronic structure which contributes to the NLO responses, the polyene to cyanine-like path is further evident from quaternary $\mathrm{N}$ as acceptor and electronrich diethyl amine $\mathrm{N}$ as donor. From both the BLA and BOA values obtained for four NLOphores, it is clear that the chromophores are more like cyanine frameworks which are the favorable structural arrangement for the compound to be highly NLO responsive. This is well reflected in the large polarizability and hyperpolarizability values obtained for these NLOphores.

Commonly used $\pi$-conjugated system in push-pull chromophores such as olefins can be replaced with linker such as five or six membered heterocyclic compounds. This provides the rigidity to a conjugated system, thus produces the effective intramolecular charge transfer, and cis-trans isomerization is reduced, which ensures a minimal loss of energy by non-radiative way. In the case of coumarin-fused BODIPY hybrids, six membered lactone ring from coumarin and quasiaromatic central ring of BODIPY core play the role as heterocyclic linker.

It was also concluded that only increasing the $\mathrm{D}$ or $\mathrm{A}$ strength does not necessarily enhance the hyperpolarizability, but it also depends upon whether the two states are fully delocalized or localized. ${ }^{24,25}$ The optimum response can be achieved at an intermediate point where there is an appreciable overlap of the wave functions that define the two states. ${ }^{22}$ Hence, an overlap between the HOMO and LUMO levels over the $\pi$-conjugated bridge is necessary to obtain large hyperpolarizability in a push-pull system. ${ }^{26,28}$ The frontier molecular orbital (FMO) images of the molecule at HOMO and LUMO present the picture of overlap. Hence, the FMO images of dyes 1 to $\mathbf{4}$ were obtained from DFT computations by B3LYP/6-311+G(d,p) method and are presented in Table 4.

As shown in Table 4 of FMO diagrams, for all the chromophores, the HOMO is localized on the donor end of the molecule and $\pi$-conjugated linker while the LUMO is localized primarily on the acceptor BODIPY 
Table 4. FMO images of dyes $\mathbf{1}$ to $\mathbf{4}$ at HOMO and LUMO level. Energy level and electron density distribution of frontier molecular orbitals of dyes 1-4 in DCM solvent, calculated by B3LYP/6 -311+G(d,p) level of theory. (The orbital diagrams are plotted with the contour value of 0.02 a. u.).

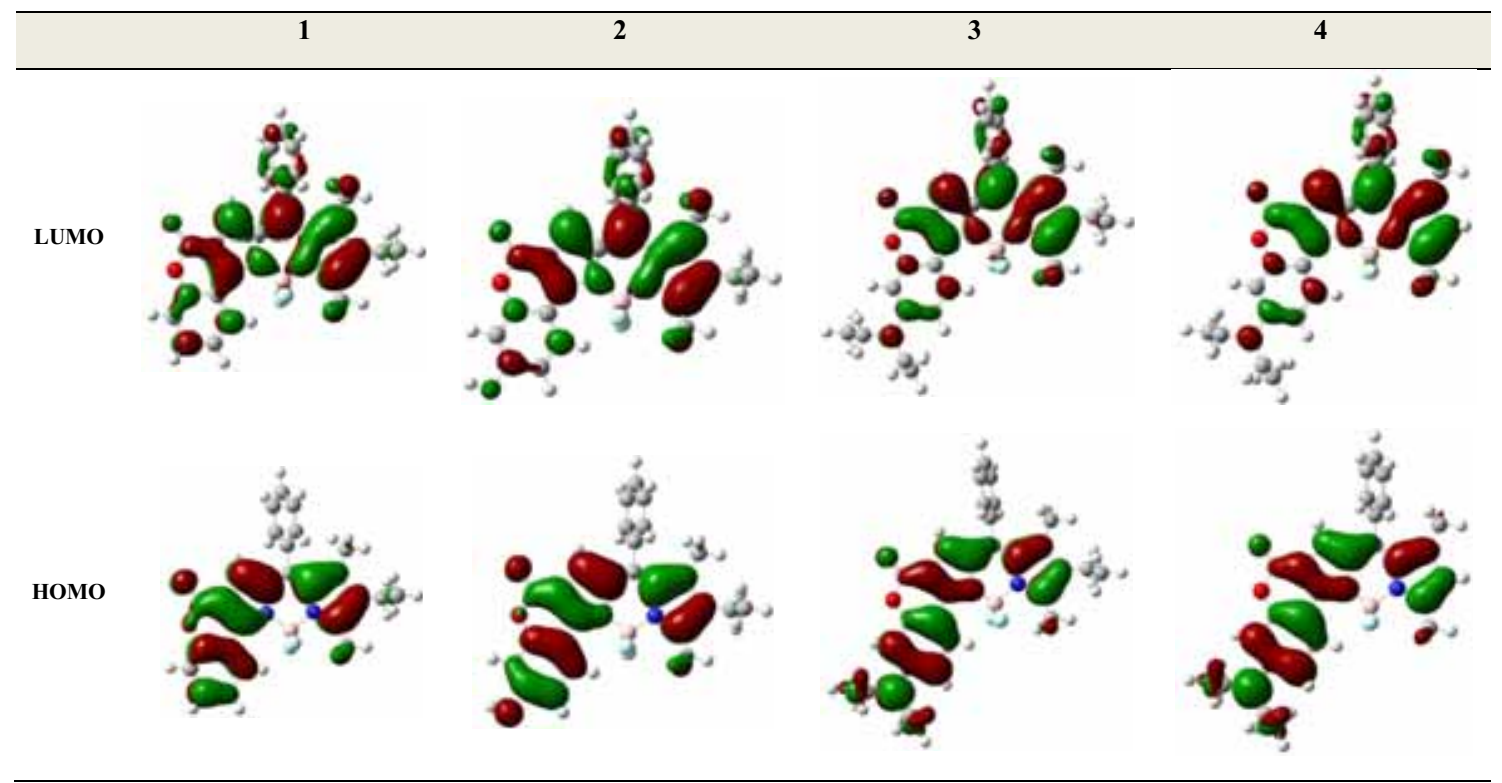

part of the molecule. This confirms that the donor part is lying on the coumarin end and acceptor part on BODIPY end. Also the delocalization of electron density over $\pi$ conjugation at HOMO and LUMO levels confirms the effective overlap of the two states to obtain maximum NLO response. These charge transfer characteristic features of the CB chromophores is the basis for NLO property study.

The common method used to measure the $\beta \mathrm{xxx}$ or $\beta C T$ values of organic NLOphores is an EFISHG (electronic field induced second harmonic generation) technique, but its experimental set up is not an easy way in most of the laboratories. ${ }^{53,54}$ Hence, the solvatochromic method to measure first order hyperpolarizability $(\beta \mathrm{xxx}$ or $\beta \mathrm{CT}$ ) has been preferred often due to its easiness. ${ }^{55}$

\section{2a Determination of first polarizability $\left(\alpha_{\mathrm{xx}}\right.$ or $\left.\alpha_{\mathrm{CT}}\right)$ :}

The NLOphores have an electron-donor group and an acceptor group linked through a $\pi$-conjugated system. Therefore, the $S_{1}$ state is frequently a low-lying chargetransfer state, hence the dominant component of the first order polarizability, often referred as $\alpha_{\mathrm{CT}}$, whose apparent expression is,

$\alpha_{C T}=\alpha_{x x}=2 \frac{\mu_{e g}^{2}}{E_{e g}}=\frac{2 \mu_{e g}^{2} \lambda_{e g}}{h c}$

where, $\mathrm{x}$ is direction of charge transfer, $h=$ Planks constant, $\mathrm{c}=$ velocity of light in vacuum, $\lambda_{e g}=$ the wavelength of transition from the ground state to excited state, $\mu_{\mathrm{eg}}$ is the transition dipole moment, which is related to the oscillator strength $f$. Using the values of eqs. 2 and 3 , the values $\alpha_{\mathrm{CT}}$ for the dyes 1-4 in DCM were calculated and summarized in Table 5. Table 5 reveals that the $\alpha_{\text {CT }}$ values of dyes are almost doubled on going from 1 and $\mathbf{2}$, to 3 and $\mathbf{4}$.

\section{2b Determination of first order hyperpolarizability} $\left(\beta_{\mathbf{C T}}\right)::$ The first order hyperpolarizability $\left(\beta_{C T}\right)$ is determined using two level microscopic model, based on the Oudar equation. ${ }^{56,57}$ The modified form of Oudar equation can be presented as,

$\beta_{C T}=\beta_{x x x}=\frac{3}{2 h^{2} C^{2}} \times \frac{\bar{v}_{e g}^{2} \mu_{e g}^{2} \Delta \mu_{C T}}{\left(v_{e g}^{2}-v_{L}^{2}\right)\left(v_{e g}^{2}-4 v_{L}^{2}\right)}$

where, $v_{L}$ is the frequency of the reference incident radiation to which the $\beta_{C T}$ value would be referred. $\nu_{e g}$ is the frequency and $\Delta \mu_{C T}$ is the dipole moment difference between the excited and ground state. Assuming $v_{L}=0$, i.e., assuming no excitation, eq. 10 simplifies to,

$\beta_{C T}=\beta_{x x x}=\frac{3}{2} \frac{\mu_{e g}^{2} \Delta \mu_{C T}}{\left(E_{e g}^{2}\right)}$

The two level microscopic model gives a rough estimation of the values of $\beta_{C T}$ as the method is based on several assumptions. Hence, it allows only estimating the leading tensor of total hyperpolarizability along the direction of charge transfer, which is the major contributor to the $\beta_{\mathrm{CT}}$. The values obtained for $\beta_{\mathrm{CT}}$ are summarized 
Table 5. Linear and nonlinear optical property data of dyes $\mathbf{1}$ to $\mathbf{4}$.

\begin{tabular}{lcccccccc}
\hline Comp. & $\mu^{[\mathrm{a}]}$ & $\begin{array}{c}\alpha_{\mathrm{CT}}^{[\mathrm{b}]} \\
/ 10^{-24} \\
\text { e.s.u }\end{array}$ & $\begin{array}{c}\alpha_{0}^{[\mathrm{c}]} \\
/ 10^{-24} \\
\text { e.s.u }\end{array}$ & $\begin{array}{c}\Delta \alpha^{[\mathrm{d}} \\
/ 10^{-24} \\
\text { e.s.u }\end{array}$ & $\begin{array}{c}\beta_{\mathrm{CT}}^{[\mathrm{e}]} \\
/ 10^{-30} \\
\text { e.s.u }\end{array}$ & $\begin{array}{c}\beta_{0}^{[\mathrm{f}]} \\
/ 10^{-30} \\
\text { e.s.u }\end{array}$ & $\begin{array}{c}\mu . \beta_{0}^{\mathrm{g}]} \\
/ 10^{-48} \\
\text { e.s.u }\end{array}$ & $\begin{array}{c}\gamma^{[\mathrm{h}]} \\
\text { e.s.u }\end{array}$ \\
\hline $\mathbf{1}$ & & & & & & & & \\
B3LYP & 10.21 & & 47.69 & 85398 & & 128.13 & 1308.18 & 391.95 \\
BHandHLYP & 10.71 & 25.67 & 45.59 & 75926 & 3.48 & 130.08 & 1392.52 & 302.93 \\
CAMB3LYP & 10.66 & & 46.1 & 77235 & & 136.54 & 1454.92 & 307.03 \\
2 & & & & & & & & \\
B3LYP & 8.23 & & 48.21 & 97467 & & 222.45 & 1830.14 & 584.82 \\
BHandHLYP & 9.01 & 25.25 & 45.92 & 83628 & 3.52 & 189.72 & 1708.7 & 404.95 \\
CAMB3LYP & 8.92 & & 46.55 & 85871 & & 201.63 & 1798.22 & 428.13 \\
3 & & & & & & & & \\
B3LYP & 6.24 & & 53.01 & 121524 & & 609.88 & 3805.91 & 1938.52 \\
BHandHLYP & 6.96 & 50.83 & 44.63 & 114450 & 0.42 & 412.79 & 2871.49 & 1168.04 \\
CAMB3LYP & 6.86 & & 45.26 & 117818 & & 431.27 & 2958.87 & 1224.68 \\
$\mathbf{4}$ & & & & & & & & \\
B3LYP & 5.17 & & 57.23 & 101414 & & 617.29 & 3191.27 & 1888.09 \\
BHandHLYP & 5.84 & 50.06 & 44.43 & 100338 & 3.42 & 419.5 & 2448.69 & 1161.46 \\
CAMB3LYP & 5.73 & & 45.06 & 103423 & & 438.3 & 2510.98 & 1216.91 \\
\hline
\end{tabular}

[a] Total static dipole moment by DFT; [b] Experimental first order polarizability; [c] first order polarizability by DFT; [d] polarization anisotropy by DFT; [e] Experimental first order hyperpolarizability. [f] First order hyperpolarizability by DFT; [g] quadratic hyperpolarizability; [h] second order hyperpolarizabilityby DFT.

in Table 5. Table 5 reveals that the dyes 1-4 possess values of the order of $10^{-30}$ e.s.u.

\subsection{NLO properties of the dyes 1-4, using TD-DFT}

The use of appropriate functional in computational strategy to calculate the molecular hyperpolarizability is highly demanding. This can be provided either by the choice of hybrid functional, which uses a fixed amount of exchange at all inerelectronic distances or a longrange corrected (LC) functional, which uses a larger fraction of generalized gradient approximation (GGA) DFT exchange at short range and larger fraction of exact exchange at long range inerelectronic distances. ${ }^{58-60}$ Further, to eliminate the problem of delocalization error for the estimation of molecular hyperpolarizabilites, it is recommended to use the hybrid GGA functionals in combination with a percentage of the local exchange replaced by the exact $\mathrm{HF}^{61}$

In this context, while applying different functionals in combination with basis set, the amount of Hartree-Fock (HF) exchange energy incorporated into each functional was varied. For larger organic chromophores, common hybrid functionals like B3LYP often perform well in predicting the excitation energies which is also important for understanding the hyperpolarizabilities. ${ }^{62,63}$ Previously, our group had efficiently utilized the B3LYP functional for hyperpolarizability calculations which uses $20 \%$ Hartree-Fock exchange. ${ }^{64}$ The
CAM-B3LYP functional (range separated-generalised gradient approximation (GGA)) has 19\% (short range) to $65 \%$ (long range) Hartree-Fock exchange while BHandHlyp fuctional (hybrid GGA) has 50\% HartreeFock exchange for both short and long range separation. ${ }^{65}$ Generally, B3LYP predicts the excitation energies reasonably well, and the range separated hybrid CAM-B3LYP predicts hyperpolarizability characteristics efficiently over the excitation energies. However, the hybrid BHandHLYP with 50\% HF exchange executes the intermediate calculations efficiently compared to the B3LYP and CAM-B3LYP functionals. ${ }^{65}$

With this background we calculated the dipole moment $(\mu)$, polarizability $(\alpha)$ and hyperpolarizability $(\beta, \gamma)$ characteristics of four hybrid NLOphores by applying B3LYP/6-311+G(d,p), CAM-B3LYP/6$311+\mathrm{G}(\mathrm{d}, \mathrm{p})$ and BHandHLYP/6-311+G(d,p) methods. To obtain converged electronic properties, like polarizabilities and hyperpolarizabilities with accuracy and efficiency, the choice of basis set is crucial. Hence, large basis set is important which includes both diffuse and polarization functions. ${ }^{66}$ Diffuse functions represent more accurately the tail portion of the atomic orbitals, which are distant from the nuclei and these are frequently used for calculation of dipole moment and hyperpolarizabilities. Polarization functions add flexibility to the basis set and effectively allow the molecular orbitals to be more asymmetric about the nucleus. Therefore, we have chosen an appropriate combination of 
diffuse functions, polarized functions and contracted Guassian 6-311+d(d,p) basis set for balanced calculations. With this knowledge we calculated the dipole moment $(\mu)$, polarizability $(\alpha)$ and hyperpolarizability $(\beta, \gamma)$ characteristics of four CB hybrid NLOphores by applying B3LYP/6-311+G(d,p), CAM-B3LYP/6$311+\mathrm{G}(\mathrm{d}, \mathrm{p})$ and BHandHLYP/6-311+G(d,p) methods. Solute-solvent interactions play important role in determining the molecular electronic properties, hence direct and indirect solvent effect must be included in the treatment when computing the hyperpolarizabilities of the NLOphores. ${ }^{42}$ Hence, the basis set is coupled with polarization continuum model (PCM) to consider the bulk solvent effect in calculation of electronic as well as structural properties. ${ }^{65,67}$

The total static dipole moment $\mu$ is expressed by following equation:

$\mu=\sqrt{\mu_{x}^{2}+\mu_{y}^{2}+\mu_{z}^{2}}$

The isotropic polarizability can be calculated from the trace of the polarization tensor:

$\alpha_{0}=\frac{\left(\alpha_{x x}+\alpha_{y y}+\alpha_{z z}\right)}{3}$

$\alpha_{x x}, \alpha_{y y}, \alpha_{z z}$ are the polarizability tensor components. Anisotropy of the polarizability $\Delta \alpha$ is expressed by,

$\Delta \alpha=2^{-1 / 2}\left[\left(\alpha_{x x}+\alpha_{y y}\right)^{2}+\left(\alpha_{z z}+\alpha_{x x}\right)^{2}+6 \alpha_{x x}^{2}\right]$

The mean/static first hyperpolarizability $\left(\beta_{0}\right)$ is expressed by,

$$
\begin{aligned}
\beta_{0}= & \left(\beta_{x}^{2}+\beta_{y}^{2}+\beta_{z}^{2}\right)^{1 / 2} \\
\beta_{0}= & {\left[\left(\beta_{x x x}+\beta_{x y y}+\beta_{x z z}\right)^{2}+\left(\beta_{y x x}+\beta_{y y y}+\beta_{y z z}\right)^{2}\right.} \\
& \left.+\left(\beta_{z x x}+\beta_{z y y}+\beta_{z z z}\right)^{2}\right]^{\frac{1}{2}}
\end{aligned}
$$

Here, $\beta_{x}, \beta_{y}$, and $\beta_{z}$ are the components of the secondorder polarizability tensor along the $\mathrm{x}, \mathrm{y}$, and $\mathrm{z}$ axes. The mean second hyperpolarizability $(\gamma)$ is expressed by,

$\gamma=\frac{1}{5}\left[\left(\gamma_{x x x x}+\gamma_{y y y y}+\gamma_{z z z z}\right)+2\left(\gamma_{x x y y}+\gamma_{y y z z}+\gamma_{z z x x}\right)\right]$

where, $\gamma_{x x x x}, \gamma_{y y y y}, \gamma_{z z z z}$ are the second order tensor components.

The values calculated for $\mu, \alpha_{0}, \Delta \alpha, \beta_{0}$ and $\gamma$ for the four dyes in

DCM using equations 12-17 for the above mentioned three different computational methods, respectively, are summarized in Table 5.

The static dipole moment obtained from TDDFT calculations decreases monotonically from dyes $\mathbf{1}$ to

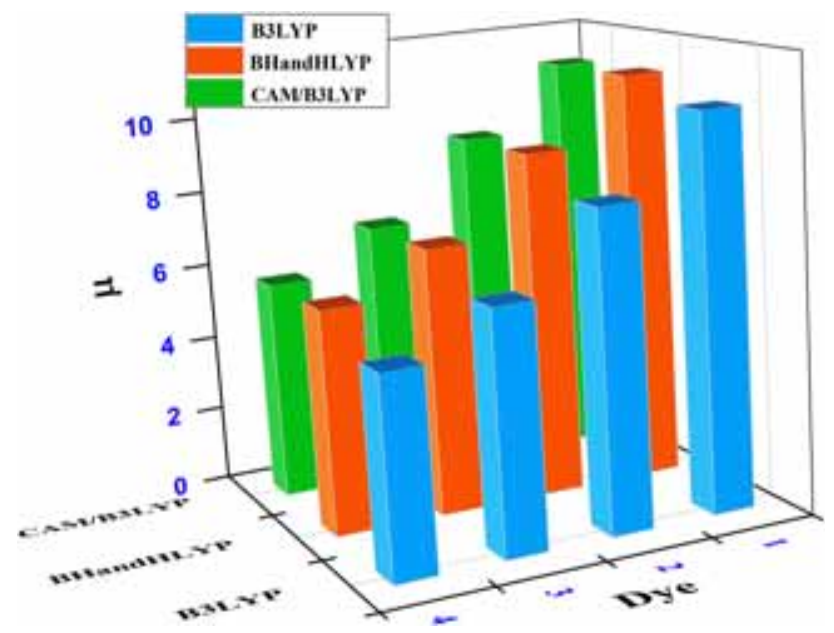

Figure 3. Static dipole moment of dyes $\mathbf{1}$ to $\mathbf{4}$ by B3LYP, CAMB3LYP, BH and HLYP.

4 and the descending values are as, BHandHLYP > CAMB3LYP $>$ B3LYP. The polarization anisotropy $(\Delta \alpha)$ values are important as it gives an account of the molecule as a photo-refractive material. Among the four dyes, dye 3 has highest values of $\alpha_{0}, \Delta \alpha$ and their respective values are decreasing for the methods as, B3LYP $>$ CAMB3LYP $>$ BHandHLYP. In general, $\beta_{0}$ values for the four dyes follow the order as, B3LYP > CAMB3LYP $>$ BHandHLYP. All the dyes exhibit very good $\beta_{0}$ values and are in the range of $10^{-30}$ e.s.u. The dye 4 shows the highest $\beta_{0}$ value among the four dyes. The three methods are compared for $\mu, \alpha_{0}$, and $\beta_{0}$ values using bar diagrams shown in Figures 3 and 4. Spectroscopic $\beta_{0}$ values are very less compared to that of the estimated by DFT method.

We have further investigated the linear and nonlinear optical properties using excited state geometry, in gas phase and in DCM, by CAM-B3LYP/6-311+g(d, p) method. The $\mu, \alpha_{0}, \beta_{0}$ and $\gamma$ values for the four dyes in GS and ES are compared in Table 6. For dyes $\mathbf{1 ,} \mathbf{3}$ and 4 , all the values of $\mu, \alpha_{0}, \beta_{0}$ and $\gamma$ in DCM are more than in gas phase, while the corresponding ES values are more than the GS values. For dye 2, the respective values in DCM are more than in gas phase but the ES and GS values have very little difference. The $1^{\text {st }}$ and $2^{\text {nd }}$ order hyperpolarizability values for dyes $\mathbf{3}$ and $\mathbf{4}$ are more than those of $\mathbf{1}$ and $\mathbf{2}$ owing to the strong ICT character arising from strong $\mathrm{N}, \mathrm{N}$-diethyl donor. The ethyl group on BODIPY part of dye $\mathbf{3}$ has a little effect on NLO response to yield slightly higher values of hyperpolarizability.

The comparative GS and ES values of $\mu, \beta_{0}$ and $\gamma$ for the four dyes are compared in bar diagrams as shown in Figures 5, 6a and $6 \mathrm{~b}$. 

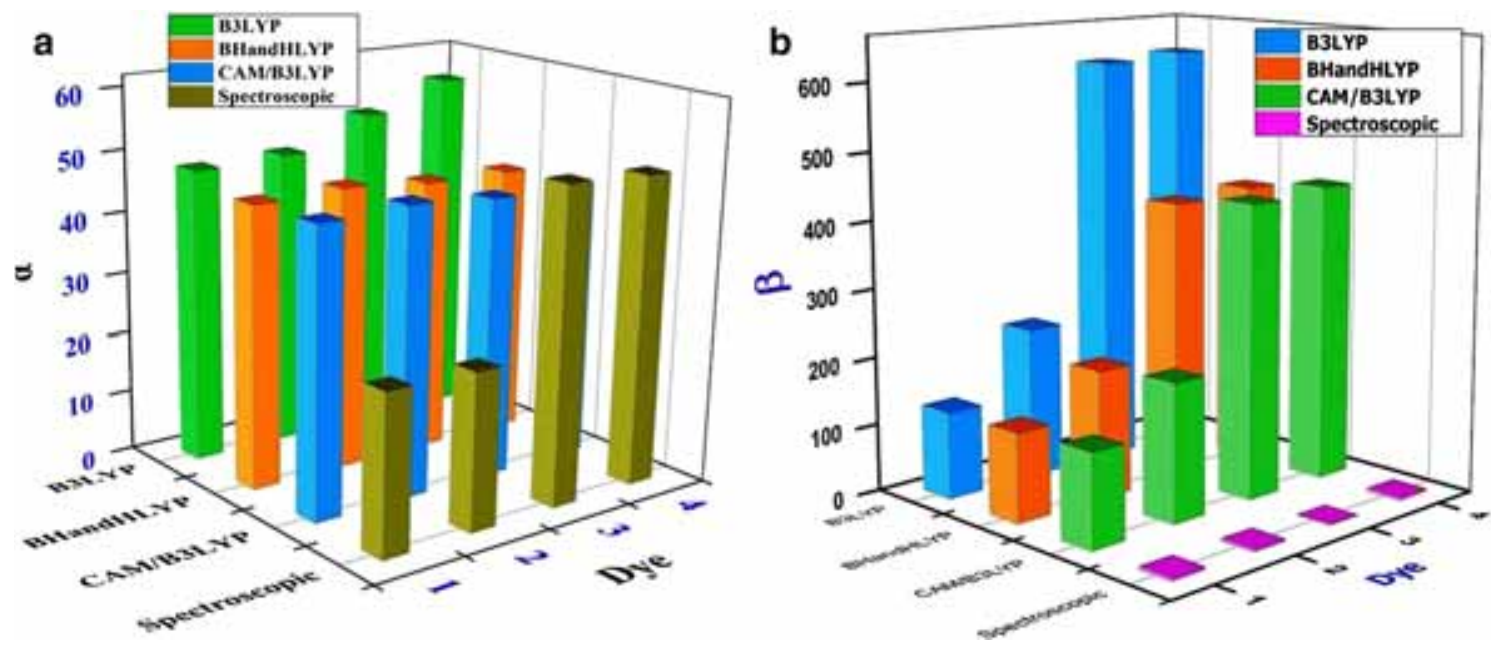

Figure 4. (a) Linear polarizability ( $\alpha$ ), and (b) First order hyperpolarizability ( $\beta$ ) of dyes 1 to 4 by B3LYP, CAMB3LYP, BHandHLYP and spectroscopic methods.

Table 6. GS and ES linear and nonlinear characteristics of dyes $\mathbf{1}$ to $\mathbf{4}$ in gas phase and DCM.

\begin{tabular}{|c|c|c|c|c|c|c|c|c|c|c|c|c|c|c|c|c|}
\hline \multirow[t]{4}{*}{ Dye } & \multicolumn{4}{|c|}{$\mu^{[\mathrm{a}]}$} & \multicolumn{4}{|c|}{$\begin{array}{c}\alpha_{0}^{[\mathrm{b}]} \\
/ 10-24 \\
\end{array}$} & \multicolumn{4}{|c|}{$\begin{array}{c}\beta_{0}^{[\mathrm{c}]} \\
/ 10^{-30}\end{array}$} & \multicolumn{4}{|c|}{$\begin{array}{c}\gamma^{[\mathrm{d}]} \\
/ 10^{-36}\end{array}$} \\
\hline & \multicolumn{4}{|c|}{ Debye } & \multicolumn{4}{|c|}{ e.s.u } & \multicolumn{4}{|c|}{ e.s.u } & \multicolumn{4}{|c|}{ e.s.u } \\
\hline & \multicolumn{2}{|c|}{ Gas } & \multicolumn{2}{|c|}{ DCM } & \multicolumn{2}{|c|}{ Gas } & \multicolumn{2}{|c|}{ DCM } & \multicolumn{2}{|c|}{ Gas } & \multicolumn{2}{|c|}{ DCM } & \multicolumn{2}{|c|}{ Gas } & \multicolumn{2}{|c|}{ DCM } \\
\hline & $\overline{\mathrm{GS}}$ & ES & GS & $\overline{E S}$ & $\mathrm{GS}$ & $\mathrm{ES}$ & GS & $\mathrm{ES}$ & GS & ES & GS & ES & GS & ES & GS & ES \\
\hline 1 & 8.1 & 10.3 & 10.7 & 10.7 & 33.9 & 32.8 & 46.1 & 46.4 & 61.2 & 81.3 & 136.5 & 167.1 & 91.7 & 130.3 & 307.0 & 354.7 \\
\hline 2 & 6.7 & 7.7 & 8.9 & 9.1 & 33.8 & 34.1 & 46.6 & 47.1 & 91.0 & 118.4 & 201.6 & 258.1 & 124.6 & 177.8 & 428.1 & 555.5 \\
\hline 3 & 4.8 & 6.2 & 6.9 & 7.6 & 35.1 & 32.6 & 45.3 & 44.6 & 185.6 & 228.9 & 431.3 & 522.0 & 339.1 & 502.9 & 1224.7 & 1650.3 \\
\hline 4 & 4.0 & 5.4 & 5.7 & 6.6 & 35.5 & 30.9 & 45.1 & 43.2 & 176.8 & 218.0 & 438.3 & 522.6 & 316.9 & 476.5 & 1216.9 & 1643.8 \\
\hline
\end{tabular}

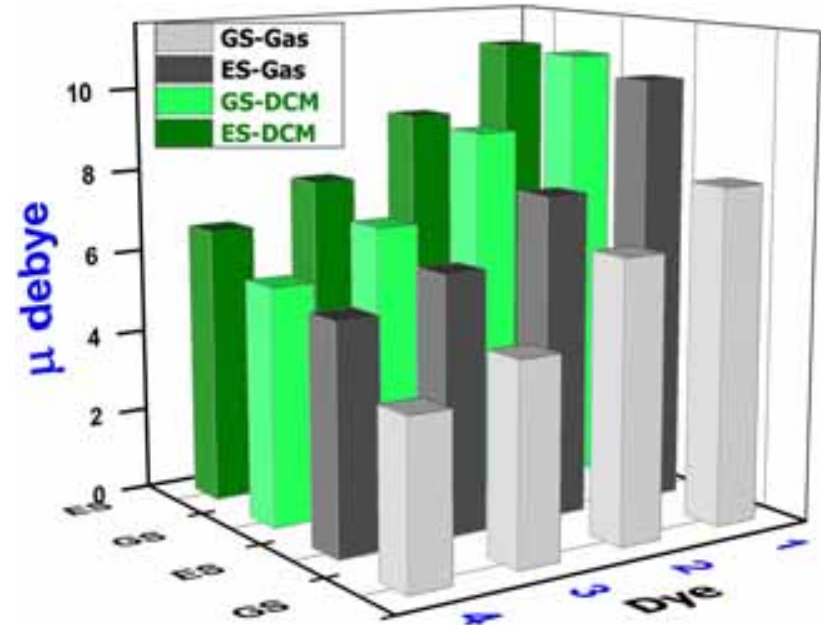

Figure 5. GS and ES static dipole moment of dyes 1 to $\mathbf{4}$ by CAM-B3LYP/6-311+G(d,p) method.

The BLA and BOA at ES and GS for the four dyes are correlated with $\beta_{0}$ values in Figures $7 \mathrm{a}$ and $7 \mathrm{~b}$, respectively. Both figures show linear correlation of $\beta_{0}$ values with BLA/BOA values in ES and GS. At ES, in case of dyes 1 to 4 , the $\beta$ value with BOA decreases and with BLA increases. While at GS, in case of dyes 1 to 4 , the $\beta$ value with BOA increases and with BLA decreases.

\subsection{Performance comparison of hybrid chromophores with their isolated units}

We have compared polarizability and hyperpolarizability values of hybrid dyes $\mathbf{1}$ to $\mathbf{4}$ with the isolated coumarin and BODIPY units (Figure 8). The NLO response of the $\mathrm{C} 1, \mathrm{C} 2, \mathrm{~B} 1$ and $\mathrm{B} 2$ was calculated at the same level of theory which was used for the hybrid chromophores; i.e., CAM-B3LYP/6-311+G(d,p). It was observed that all hybrid chromophores, particularly dyes 3 and 4, show comparatively very high NLO response than either of the isolated units (Table 7). This justifies the designing and investigation strategy applied to study the NLO response of coumarin-fused BODIPY hybrids.

The hybrid dyes $\mathbf{1}$ to $\mathbf{4}$ have positive values for static second order hyperpolarizability $\gamma$, while this is not the case for the constituents of hybrid structures, namely, 

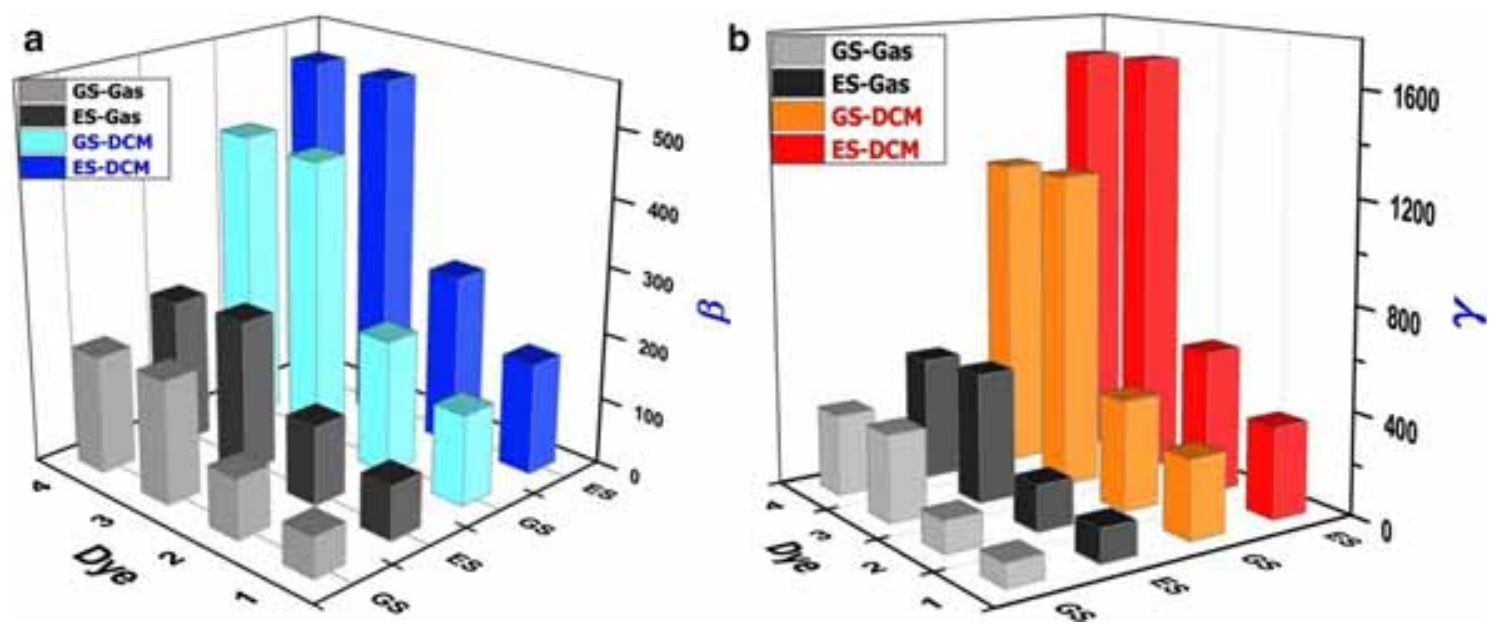

Figure 6. (a) first order hyperpolarizability and (b) second order hyperpolarizability of dyes 1 to 4 in GS and ES by CAM-B3LYP/6-311+G(d,p) method.
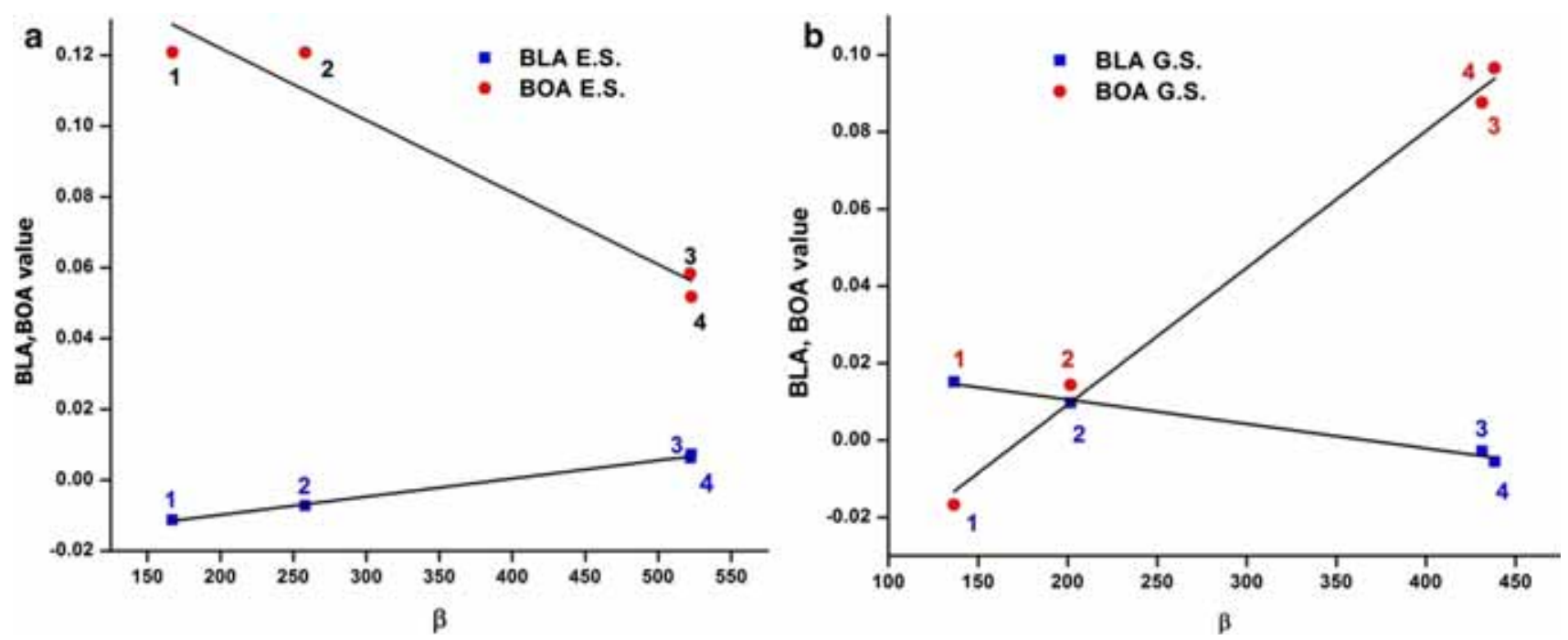

Figure 7. (a) Plot of $\beta$ vs BLA/BOA (ES); (b) Plot of $\beta$ vs BLA/BOA (GS).<smiles>O=c1ccc2ccc(O)cc2o1</smiles>

C1<smiles>CCN(CC)c1ccc2ccc(=O)oc2c1</smiles>

$\mathrm{C} 2$<smiles>Cc1cc(C)n(-c2ccccc2)c1-c1cccn1P</smiles>

B1<smiles>CCC1=C(C)C2=C(c3ccccc3)c3cccn3B(F)[N+]2=C1C</smiles>

B2

Figure 8. Structures of isolated coumarin and BODIPY units.

coumarin and BODIPY. The coumarins $\mathrm{C} 1$ and $\mathrm{C} 2$ have positive $\gamma$ values while the BODIPYs B1 and B2 have negative $\gamma$. It is known that when the system has the capacity to exhibit anion radical character ${ }^{68}$ or large quinoidal character, ${ }^{69}$ the $\gamma$ value tends to be negative. One may presume that such characters leading to a large transition dipole moments are responsible for the negative $\gamma$ values in BODIPYs B1 and B2. When both the systems are conjugatively fused as in the hybrids $\mathbf{1}$ to 4 , the value of $\gamma$ is no longer negative. It is likely that tunability of $\gamma$ can be achieved by hybridisation of two different chromophores. 
Table 7. Comparison of polarizability and hyperpolarizability values of C1, C2, B1, B2 with dyes 1 to 4 .

\begin{tabular}{lcrrrrrrr}
\hline Dye & 1 & 2 & 3 & 4 & $\mathrm{C} 1$ & $\mathrm{C} 2$ & $\mathrm{~B} 1$ & $\mathrm{~B} 2$ \\
\hline$\alpha_{0}$ & 46.1 & 46.55 & 45.26 & 45.06 & 15.7 & 16.02 & 65.02 & 39.01 \\
$\beta_{0}$ & 130.08 & 201.63 & 431.27 & 438.3 & 16.388 & 48.62 & 346.57 & 360.12 \\
$\gamma$ & 302.93 & 428.13 & 1224.68 & 1216.91 & 33.65 & 93.95 & -357.72 & -318.45 \\
\hline$\alpha_{0}\left(\times 10^{-24}\right)$ esu, $\beta_{0}\left(\times 10^{-30}\right)$ esu, $\gamma\left(\times 10^{-36}\right)$ esu. & & &
\end{tabular}

\section{Conclusions}

The NLO properties of coumarin fused BODIPY chromophores were investigated in details. Spectroscopic method gives rough estimation of polarizability and hyperpolarizability characteristics of four hybrid chromophores while by computational approach three different hybrid functionals B3LYP, CAM-B3LYP and BHandHLYP in combination with $6-311+\mathrm{G}(\mathrm{d}, \mathrm{p})$ basis set gives comparative estimation of these values. B3LYP slightly overestimates the NLO responses as compared to the other two functionals. NLO responses arising from the excited state geometries are better, comparable to the ground state obtained from theoretical method. Further GMH analysis account the charge transfer characteristics of the chromophores and BLA-BOA calculations at ground and excited state suggests that the cyanine type $D-\pi$-A framework resulting in high NLO response. The hybrid molecules are proved to be the better performing NLOphores than their individual isolated coumarin or BODIPY cores. Thus with an added advantage of their improved absorption and emission properties, the hybrid chromophore comes out as a new promising class of NLO materials over the coumarin and BODIPY units.

\section{Supplementary Information (SI)}

Cartesian coordinates of dyes $\mathbf{1}$ to $\mathbf{4}$ for optimized geometry at B3LYP/6-311+G(d,p) level in dichloromethane solvent are available at www.ias.ac.in/chemsci.

\section{Acknowledgements}

YE gratefully acknowledges the financial support from the UGC, New Delhi, Govt. of India for SRF fellowship, file number F.4-1/2006(BSR)/8-10/2007(BSR).

\section{References}

1. Liu S, Shi Z, Xu W, Yang H, Xi N, Liu X, Zhao Q and Huang W 2014 A Class of Wavelength-Tunable nearInfrared Aza-BODIPY Dyes and Their Application for Sensing Mercury Ion Dyes Pigm. 103145
2. Zheng Q, He G S and Prasad P N 2009 A Novel near IR Two-Photon Absorbing Chromophore: Optical Limiting and Stabilization Performances at an Optical Communication Wavelength Chem. Phys. Lett. 475250

3. Kubo Y, Eguchi D, Matsumoto A, Nishiyabu R, Yakushiji H, Shigaki K and Kaneko M 2014 BoronDibenzopyrromethene-Based Organic Dyes for Application in Dye-Sensitized Solar Cells J. Mater. Chem. A 25204

4. Baruah M, Qin W, Vallée R A L, Beljonne D, Rohand T, Dehaen W and Boens N A 2005 Highly PotassiumSelective Ratiometric Fluorescent Indicator Based on BODIPY Azacrown Ether Excitable with Visible Light Org. Lett. 74377

5. Sheik-Bahae M, Said A A, Wei T H, Hagan D J and Van Stryland E W 1990 Sensitive Measurement of Optical Nonlinearities Using a Single Beam IEEE J. Quantum Electron. 26760

6. Chibani S, Laurent A D, Le Guennic B and Jacquemin D 2014 Improving the Accuracy of Excited-State Simulations of BODIPY and Aza-BODIPY Dyes with a Joint SOS-CIS(D) and TD-DFT Approach J. Chem. Theory Comput. 104574

7. Jiang X D, Li S, Le Guennic B, Jacquemin D, Escudero D and Xiao L 2016 Singlet Oxygen Generation Properties of Isometrically Dibromated Thienyl-Containing AzaBODIPYs Phys. Chem. Chem. Phys. 1832686

8. Azarias C, Russo R, Cupellini L, Mennucci B and Jacquemin D 2017 Modeling Excitation Energy Transfer in Multi-BODIPY Architectures Phys. Chem. Chem. Phys. 196443

9. Chibani S, Le Guennic B, Charaf-Eddin A, Laurent A D and Jacquemin D 2013 Revisiting the Optical Signatures of BODIPY with Ab Initio Tools Chem. Sci. 4 1950

10. Acebal P, Blaya S and Carretero L 2003 Dipyrromethene-BF2 Complexes with Optimized Electrooptic Properties Chem. Phys. Lett. 382489

11. Zhu M, Jiang L, Yuan M, Liu X, Ouyang C, Zheng H, Yin X, Zuo Z, Liu H and Li Y 2008 Efficient Tuning Nonlinear Optical Properties: Synthesis and Characterization of a Series of Novel Poly(aryleneethynylene)s Co-Containing BODIPY J. Polym. Sci. Part A Polym. Chem. 67401

12. Shi W J, Lo P C, Singh A, Ledoux-Rak I and Ng D K P 2012 Synthesis and Second-Order Nonlinear Optical Properties of Push-Pull BODIPY Derivatives Tetrahedron 688712

13. Frenette M, Hatamimoslehabadi M, Bellinger-Buckley S, Laoui S, Bag S, Dantiste O, Rochford $\mathrm{J}$ and 
Yelleswarapu C 2014 Nonlinear Optical Properties of Multipyrrole Dyes Chem. Phys. Lett. 608303

14. Bouit P A, Kamada K, Feneyrou P, Berginc G, Toupet L, Maury O and Andraud C 2009 Two-Photon AbsorptionRelated Properties of Functionalized BODIPY Dyes in the Infrared Range up to Telecommunication Wavelengths Adv. Mater. 211151

15. Didier P, Ulrich G, Mély Y and Ziessel R 2009 Improved Push-Pull-Push E-Bodipy Fluorophores for Two-Photon Cell-Imaging Org. Biomol. Chem. 73639

16. Zhang D, Wang Y, Xiao Y, Qian S and Qian X 2009 Long-Wavelength Boradiazaindacene Derivatives with Two-Photon Absorption Activity and Strong Emission: Versatile Candidates for Biological Imaging Applications Tetrahedron $\mathbf{6 5} 8099$

17. Liu B, Li L, Lin C, Zhou J, Zhu Z, Xu H, Qiu H and Yin S 2014 Polyacetylenes Containing BODIPY Pendants with Different Connectivities: Synthesis, Characterization and Opto-Electronic Properties Polym. Chem. 5372

18. Ward J F 1965 Calculation of Nonlinear Optical Susceptibilities Using Diagrammatic Perturbation Theory Rev. Mod. Phys. 371

19. Oudar J L 1977 Optical Nonlinearities of Conjugated Molecules. Stilbene Derivatives and Highly Polar Aromatic Compounds J. Chem. Phys. 67446

20. Oudar J L and Le Person H 1975 Second-Order Polarizabilities of Some Aromatic Molecules Opt. Commun. $\mathbf{1 5} 258$

21. LeCours S M, Guan H W, DiMagno S G, Wang C $\mathrm{H}$ and Therien M J 1996 Push-Pull Arylethynyl Porphyrins: New Chromophores That Exhibit Large Molecular First-Order Hyperpolarizabilities J. Am. Chem. Soc. 1181497

22. Kanis D R, Ratner M A and Marks T J 1994 Design and Construction of Molecular Assemblies with Large Second-Order Optical Nonlinearities. Quantum Chemical Aspects Chem. Rev. 94195

23. Zyss J 1982 New Organic Molecular Materials for Nonlinear Optics J. Non-Cryst. Solids 47211

24. Marder S R, Kippelen B, Jen A K Y and Peyghambarian N 1997 Design and Synthesis of Chromophores and Polymers for Electro-Optic and Photorefractive Applications Nature 388845

25. Zerner M C, Fabian WM F, Dworczak R, Kieslinger D W, Kroner G, Junek H and Lippitsch M E 2000 Nonlinear Optical Properties of Dicyanomethylene-Derived Heteroaromatic Dyes: Semiempirical Molecular Orbital Calculations and Experimental Investigations Int. J. Quantum Chem. 79253

26. Yoshimura T 1989 Enhancing Second-Order Nonlinear Optical Properties by Controlling the Wave Function in One-Dimensional Conjugated Molecules Phys. Rev. B 406292

27. Yoshimura T 1990 Design and Evaluation of Organic Nonlinear Optical Materials with a Large Pockels Effect Mol. Cryst. Liq. Cryst. Inc. Nonlinear Opt. 18243

28. Yoshimura T 1989 Theoretically Predicted Influence of Donors and Acceptors on Quadratic Hyperpolarizabilities in Conjugated Long-Chain Molecules Appl. Phys. Lett. 55534

29. Zyss J and Ledoux I 1994 Nonlinear Optics in Multipolar Media: Theory and Experiments Chem. Rev. 9477
30. Marder S R, Gorman C B, Tiemann B G, Perry J W, Bourhill G and Mansour K 1993 Relation between Bond-Length Alternation and Second Electronic Hyperpolarizability of Conjugated Organic Molecules Science 261186

31. Meyers F, Marder S R, Pierce B M and Bredas J L 1994 Electric Field Modulated Nonlinear Optical Properties of Donor-Acceptor Polyenes: Sum-Over-States Investigation of the Relationship between Molecular Polarizabilities (.alpha., .beta., and .gamma.) and Bond Length Alternation J. Am. Chem. Soc. 11610703

32. Albert I D L, Marks T J and Ratner M A 1997 Large Molecular Hyperpolarizabilities. Quantitative Analysis of Aromaticity and Auxiliary Donor-Acceptor Effects $J$. Am. Chem. Soc. 1196575

33. Albert I D L, Marks T J and Ratner M A 1998 Large Molecular Hyperpolarizabilities in "Push-Pull" Porphyrins. Molecular Planarity and Auxiliary DonorAcceptor Effects Chem. Mater. 10753

34. Baojun Li, Guozheng Li, Enke Liu, Zuimin J, Chengwen P and X W 1999 1.55 Mm Reflection-Type Optical Waveguide Switch Based on SiGe/Si Plasma Dispersion Effect Appl. Phys. Lett. 751

35. Suresh S, Ramanand A, Jayraman D and Mani P 2012 Review on Theoretical Aspect of Nonlinear Optics Rev. Adv. Mat. Sci. 30175

36. Bochkov A Y, Akchurin I O, Dyachenko O A and Traven V F 2013 NIR-Fluorescent Coumarin-Fused BODIPY Dyes with Large Stokes Shifts Chem. Commun. 4911653

37. Gieseking R L, Risko C and Brédas J L 2015 Distinguishing the Effects of Bond-Length Alternation versus Bond-Order Alternation on the Nonlinear Optical Properties of $\pi$-Conjugated Chromophores J. Phys. Chem. Lett. 62158

38. Sharma P K and Singh N 1969 Mean Square Amplitude of Thermal Vibrations in Cubic Metals at Melting Point Chem. Phys. Lett. 41

39. Treutler O and Ahlrichs R 1995 Efficient Molecular Numerical Integration Schemes J. Chem. Phys. 102346

40. Becke A D 1993 Density-Functional Thermochemistry. III. The Role of Exact Exchange J. Chem. Phys. 985648

41. Lee C, Yang W and Parr R G 1988 Development of the Colle-Salvetti Correlation-Energy Formula into a Functional of the Electron Density Phys. Rev. B 37 785

42. Benassi E, Egidi F and Barone V 2015 General Strategy for Computing Nonlinear Optical Properties of Large Neutral and Cationic Organic Chromophores in Solution J. Phys. Chem. B 1193155

43. Wong M W, Frisch M J and Wiberg K B 1991 Solvent Effects. 1. The Mediation of Electrostatic Effects by Solvents J. Am. Chem. Soc. 1134776

44. Frisch M J et al., 2010 Gaussian 09 Revision C01 (Wallingford: Gaussian Inc.)

45. Dennington R, Keith $\mathrm{T}$ and Millam J GaussView 5 citation. http://www.gaussian.com/g_tech/gv5ref/ gv5citation.htm (Accessed date: 10 February 2017)

46. Coe B J, Harris J A, Asselberghs I, Clays K, Olbrechts G, Persoons A, Hupp J T, Johnson R C, Coles S J, Hursthouse M B and Nakatani K 2002 Quadratic Nonlinear Optical Properties of N-Aryl Stilbazolium Dyes $A d v$. Funct. Mater. 12110 
47. Turro N J 1978 In Modern Molecular Photochemistry (New York: Benjamin-Cummings)

48. Coe B J, Fielden J, Foxon S P, Harris J A, Helliwell M, Brunschwig B S, Asselberghs I, Clays K, Garín J and Orduna J 2010 Diquat Derivatives: Highly Active, TwoDimensional Nonlinear Optical Chromophores with Potential Redox Switchability J. Am. Chem. Soc. 132 10498

49. Shawn M, Abernathy and Robert R 1997 SharpView Spin Dynamics Calculations of Electron and Nuclear Spin Relaxation Times in Paramagnetic Solutions $J$. Chem. Phys. 1069032

50. Creutz C, Newton M D and Sutin N 1994 Metal—lingad and Metal-metal Coupling Elements J. Photochem. Photobiol. A Chem. 8247

51. Rust M, Lappe J and Cave R J 2002 Multistate Effects in Calculations of the Electronic Coupling Element for Electron Transfer Using the Generalized Mulliken-Hush Method J. Phys. Chem. A 1063930

52. Newton M D 1997 Medium Reorganization and Electronic Coupling in Long-Range Electron Transfer $J$. Electroanal. Chem. 4383

53. Bethea C G 1975 Experimental Technique of Dc Induced SHG in Liquids: Measurement of the Nonlinearity of CH2I2 Appl. Opt. 141447

54. Bosshard C, Knöpfle G, Prêtre P and Gunter P 1992 Second-order Polarizabilities of Nitropyridine Derivatives Determined with Electric-field-induced Secondharmonic Generation and a Solvatochromic Method: A Comparative Study J. Appl. Phys. 711594

55. Paley M S, Harris J M, Looser H, Baumert J C, Bjorklund G C, Jundt D and Twieg R J 1990 A Solvatochromic Method for Determining Second-Order Polarizabilities of Organic Molecules [Erratum to Document Cited in CA111(11):96600e] J. Org. Chem. 551692

56. Oudar J L and Chemla D S 1997 Hyperpolarizabilities of the Nitroanilines and Their Relations to the Excited State Dipole Moment J. Chem. Phys. 662664

57. Carlotti B, Flamini R, Kikaš I, Mazzucato U and Spalletti A 2012 Intramolecular Charge Transfer, Solvatochromism and Hyperpolarizability of Compounds Bearing Ethenylene or Ethynylene Bridges Chem. Phys. 4079

58. Tawada Y, Tsuneda T, Yanagisawa S, Yanai T and Hirao K 2004 A Long-Range-Corrected Time-Dependent Density Functional Theory J. Chem. Phys. 1208425
59. Vydrov O A, Heyd J, Krukau A V and Scuseria G E 2006 Importance of Short-Range versus Long-Range HartreeFock Exchange for the Performance of Hybrid Density Functionals J. Chem. Phys. 12574106

60. Iikura H, Tsuneda T, Yanai T and Hirao K 2001 A LongRange Correction Scheme for Generalized-GradientApproximation Exchange Functionals J. Chem. Phys. 1153540

61. Cohen A J, Mori-Sánchez P and Yang W 2012 Challenges for Density Functional Theory Chem. Rev. 112 289

62. Andzelm J, Rinderspacher B C, Rawlett A, Dougherty J, Baer R and Govind N 2009 Performance of DFT Methods in the Calculation of Optical Spectra of TCF-Chromophores J. Chem. Theory Comput. 5 2835

63. Jacquemin D, Perpète E A, Scuseria G E, Ciofini I and Adamo C 2008 TD-DFT Performance for the Visible Absorption Spectra of Organic Dyes: Conventional versus Long-Range Hybrids J. Chem. Theory Comput. 4 123

64. Lanke S K and Sekar N 2015 Rigid Coumarins: A Complete DFT, TD-DFT and Non Linear Optical Property Study J. Fluoresc. 251469

65. Johnson L E, Dalton L R and Robinson B H 2014 Optimizing Calculations of Electronic Excitations and Relative Hyperpolarizabilities of Electrooptic Chromophores Acc. Chem. Res. 473258

66. Feller D and Davidson E R 1990 Basis Sets for Ab Initio Molecular Orbital Calculations and Intermolecular Interactions In Reviews in Computational Chemistry Vol. $1 \mathrm{~K}$ B Lipkowitz and D B Boyd (Eds.) (Hoboken, NJ, USA: John Wiley)

67. Tomasi J, Mennucci B and Cammi R 2005 Quantum Mechanical Continuum Solvation Models Chem. Rev. 1052999

68. Nakano M, Kiribayashi S, Yamada S and Shigemoto I 1996 Theoretical Study of the Second Hyperpolarizabilities of Three Charged States of Pentalene. A Consideration of the Structure-Property Correlation for the Sensitive Second Hyperpolarizability Chem. Phys. Lett. 26266

69. Yang M and Jiang Y 2001 Structure-Property Correlation in Static Electronic Second-Order Hyperpolarizabilities of Centrosymmetric Squaraines Chem. Phys. 274 121 